\title{
The Discoveries of Rare Pion Decays at the CERN Synchrocyclotron
}

\author{
Giuseppe Fidecaro \\ CERN, CH-1211 Geneva 23, Switzerland \\ giuseppe.fidecaro@cern.ch
}

\begin{abstract}
In 1957 the CERN $600 \mathrm{MeV}$ Synchrocyclotron started to operate and could detect for the first time already in 1958, and later in 1962, the two still missing $\beta$-decays of the charged pion, providing crucial verifications of the universal $\mathrm{V}-\mathrm{A}$ coupling.
\end{abstract}

\section{Introduction}

In October 1955, one year after its birth, CERN was exclusively a building site, where the foundations of the two CERN accelerators, the $25 \mathrm{GeV}$ Proton Synchrotron (PS) and the $600 \mathrm{MeV}$ Synchrocyclotron (SC) were laid down and the first buildings were still under construction. In the middle of 1956 the construction of the synchrocyclotron was fairly advanced and experimental physicists started gathering in the SC Division. In 1957, when the CERN synchrocyclotron started accelerating protons, and the first buildings were ready to offer office space to physicists still living in the wooden barracks at the Geneva airport site, the SC Division was already an active and lively point of attraction for people from Europe and the U.S. interested in working at the SC, and for numerous visiting physicists from other countries in the World.

Benefitting from experience at other laboratories, physicists and engineers at the SC began developing and building the first elements of experimental equipment, such as scintillation counters, all kinds of electronic circuits, and even a "synchrocyclotron simulator" to test electronic circuits in conditions similar to those encountered at the SC. ${ }^{1}$ A very helpful electronic instrument was a pulse generator based on a mercury switch relay driven by the local A.C. $50 \mathrm{~Hz}$ network, widely used in U.S. laboratories but unknown in Europe. Built by Oreste Piccioni, a visiting professor at CERN from Brookhaven in 1957, this pulse generator turned out to be invaluable for nanosecond work.

In June 1957 the engineers were getting ready to start acceleration. With the agreement of the SC engineers, in collaboration with Tito Fazzini, I installed a scintillation counter in the synchrocyclotron hall on July 16 to detect background radiation, and a rate meter with a pen recorder in the nearby experimental hall. A steady counting rate was obtained on August 1, 1957, the official date of the 
first CERN SC operation. The event was recorded in a sheet signed by Wolfgang Gentner, Head of the Division, and by all people present including Tito Fazzini, Alec Merrison and myself. We were the first physicists to see particles accelerated at CERN. A number of very interesting results were obtained in the following years, among which the two most important ones are reported in this article.

\section{Universal Fermi Interaction and Pion Decay: Two Parallel Tales}

\subsection{The weak interaction before the $\pi$-meson discovery}

After Fermi proposed in 1934 his $\beta$-decay theory ${ }^{2}$ which involved the direct emission of a pair of light particles, an electron and a neutrino, Yukawa put forward in 1935 his theory ${ }^{3}$ of exchange nuclear forces induced by emission and absorption of an intermediate particle, charged or neutral, that Yukawa named meson. There was, however, a disagreement between Fermi's and Yukawa's theories. In Yukawa's theory the electron-neutrino pair originated from the decay of the intermediate meson, while Fermi's theory was based on the direct emission of an electron-neutrino pair, with no intermediate particle at all.

In 1936, the discovery in cosmic rays of a charged particle having the mass predicted by Yukawa, between that of the electron and that of the proton, brought full support to his theory. The new cosmic-ray particle, named mesotron, was identified with Yukawa's meson, and the mesotron interaction became known as Yukawa interaction. The mesotron was recognised as the strong interaction carrier.

In 1940 it was known, as suggested by Møller, Rosenfeld and Rozenthal, ${ }^{4}$ that there were two kinds of cosmic-ray mesons, one with a mean life of the order of $10^{-8} \mathrm{~s}$, the other with a mean life of the order of $10^{-6} \mathrm{~s}$. In the same year Sakata, ${ }^{5}$ in an attempt to understand the mean lives of these mesons, described the meson decay as a compound of the Yukawa interaction $\mathrm{N} \leftrightarrow \mathrm{P}+\Pi^{-}$with the original Fermi $\beta$-decay interaction $\mathrm{N} \leftrightarrow \mathrm{P}+\mathrm{e}^{-}+\bar{\nu}$, namely $\Pi^{-} \rightarrow \overline{\mathrm{P}}+\mathrm{N} \rightarrow \mathrm{e}^{-}+\bar{\nu}$, and the charge-conjugate reaction for $\Pi^{+}$. Here the Fermi notation $\Pi$ has been used for the Yukawa meson ${ }^{6}$ (the $\pi$-meson had not been discovered yet).

While discussions on the Fermi's and Yukawa's theories were going on, a wealth of information was being accumulated from experimental work on nuclear $\beta$-decay and cosmic rays. In 1947, Conversi, Pancini and Piccioni ${ }^{7}$ discovered in Rome that negative mesotrons in the cosmic radiation coming to rest in carbon were not captured by nuclei, but they decayed into an electron and a neutral particle, either a neutrino or a $\gamma$-ray, just like positive mesotrons do.

In the same year, Pontecorvo ${ }^{8}$ was the first to notice that the capture rate of a bound negative meson $\left(\sim 10^{6} \mathrm{~s}^{-1}\right)$ is of the order of the rate of ordinary K-capture processes, when allowance is made for the difference in the disintegration energy and the difference in the volumes of the K-shell and of the meson orbit. Thus he called attention to the possible equality of the coupling constants of electrons and 
mesons (or mesotrons) to nucleons, and essentially laid down the first two sides of the Puppi triangle, ${ }^{9}$ a graphic representation of the universal weak interaction invented by J. Tiomno.

In the following years, Pontecorvo's idea developed through the work of other authors (Clementel-Puppi, O. Klein, Lee-Rosenbluth-Yang, Leite Lopes, MartyPrentki, Puppi, Tiomno-Wheeler) into the more general idea of a Universal Fermi Interaction. Namely, the various weak processes are "universal" in the sense that they are different manifestations of a single fundamental interaction (Sakurai, 1964). The name "Universal Fermi Interaction" was coined in 1950 by Yang and Tiomno. ${ }^{10}$

\subsection{The weak interaction after the $\pi$-meson discovery}

The true $\pi$-meson had been discovered in cosmic rays at Bristol in $1947^{11}$ in nuclear emulsions and detected at the Berkeley synchrocyclotron in the following year by Gardner and Lattes. ${ }^{12}$ Two examples of $\pi$-meson $\beta$-decay had been published by the Bristol group, but ... surprise! the decay products were not electrons but a new type of meson that was named $\mu$. Electrons were neither found in $\pi$-decay at the Berkeley synchrocyclotron by Bishop, Burfening, Gardner and Lattes ${ }^{13}$ nor by Lattes ${ }^{14}$ in nuclear emulsion experiments.

The absence of electron decays became a mystery, since among physicists the idea was developing that the Fermi coupling constants between Dirac particles might all be equal. For example, in the case of the following three interactions discussed by Fermi in his lectures at Yale in April 1950: ${ }^{6}$

$$
\begin{array}{cl}
\mathrm{N} \rightarrow \mathrm{P}+\mathrm{e}+\bar{\nu} & (\beta \text { decay }), \\
\mu \rightarrow \mathrm{e}+\nu+\bar{\nu} & (\mu \text { decay }), \\
\mathrm{P}+\mu^{-} \rightarrow \mathrm{N}+\nu & \left(\mu^{-} \text {capture }\right) .
\end{array}
$$

Tiomno and Wheeler, ${ }^{15}$ Lee, Rosenbluth and Yang ${ }^{16}$ and other authors had found close equality of the coupling constants, though the errors were not negligible.

However, because the decay $\pi-\mathrm{e} \nu$ had never been observed the equality of the coupling constants of the above interactions (1), (2), (3) was not sufficient proof of existence of a Universal Fermi Interaction.

In 1949, Ruderman and Finkelstein ${ }^{17}$ computed the ratio of the $\pi-\mathrm{e} \nu$ to the $\pi-$ $\mu \nu$ decay rate for various types of mesons and Fermi couplings and pointed out that, while no conclusion could be drawn on absolute rates, this ratio was independent of divergent integrals and was only a function of the pion, electron and muon masses:

$$
\begin{array}{cc}
R=\frac{\Gamma(\pi \rightarrow \mathrm{e} \nu)}{\Gamma(\pi \rightarrow \mu \nu)}=\left(\frac{M_{\pi}^{2}-M_{\mathrm{e}}^{2}}{M_{\pi}^{2}-M_{\mu}^{2}}\right)^{2} \times \frac{M_{\mathrm{e}}^{2}}{M_{\mu}^{2}}=1.28 \times 10^{-4} \quad \text { (pseudovector coupling) }, \\
R=\frac{\Gamma(\pi \rightarrow \mathrm{e} \nu)}{\Gamma(\pi \rightarrow \mu \nu)}=\left(\frac{M_{\pi}^{2}-M_{e}^{2}}{M_{\pi}^{2}-M_{\mu}^{2}}\right)^{2}=5.49 & \text { (pseudoscalar coupling). }
\end{array}
$$


In the case of vector, scalar and tensor interaction both $\pi-\mu \nu$ and $\pi-\mathrm{e} \nu$ decays are forbidden.

In 1949, Steinberger ${ }^{18}$ also made an attempt to compute the decay rates for various types of mesons and couplings using a subtraction method proposed by Pauli and Villars to deal with divergences. If one calculates $R$ from the decay rates so obtained, one gets the same result as Ruderman and Finkelstein.

Anyhow, a definite prediction for the value of $R$ existed and, according to Ruderman and Finkelstein, any theory which coupled $\pi$-mesons to nucleons also predicted $\pi-\mathrm{e} \nu$ decay. However, this decay had not been observed. Was it simply because it was a rare event in comparison with $\pi-\mu \nu$ decay?

On the one side, it was important to start a systematic search for $\pi$-e $\nu$ decay. On the other side, in the years around late 1940s and early 1950s, Scalar (S) and Tensor ( $\mathrm{T}$ ) couplings were favoured to describe the weak interaction responsible for nuclear $\beta$-decay. In such a case, according to $\mathrm{Wu}$ and Muszowski, ${ }^{19}$ the absence of $\pi-\mathrm{e} \nu$ decay did not concern physicists too much, because only two of the five possible couplings, namely A and $\mathrm{P}$, can be formed out of the pseudoscalar pion field and one four-vector, representing the non-local nature of the intermediate state. Therefore, $\mathrm{A}$ and $\mathrm{P}$ couplings are the only ones that can induce the decay of pseudoscalar pion; the other three $(\mathrm{S}, \mathrm{V}, \mathrm{T})$ are forbidden. If both $\mathrm{A}$ and $\mathrm{P}$ are lacking in $\pi$-meson decay, then $\pi-\mathrm{e} \nu$ is naturally forbidden.

Frota-Pessôa and Margem ${ }^{20}$ were the first to search for $\pi-\mathrm{e} \nu$ decay in emulsions exposed at Berkeley and found none out of $200 \pi-\mu \nu$ decays $\left(R<0.5 \times 10^{-2}\right)$. Then, in $1951 \mathrm{Smith}^{21}$ analysed emulsions that was also exposed at Berkeley and found $R=(0.3 \pm 0.4) \times 10^{-2}$ (". . less than $1 \%$ and probably zero ...").

The most sensitive emulsion experiment was done by Friedman and Rainwater who published their final results in $1951 .^{22}$ In the nuclear emulsions exposed at the Nevis synchrocyclotron, they found " ... one or zero $\pi$-e events compared to 1419 $\pi-\mu$ events $\ldots\left(R \leq 7 \times 10^{-4}\right)$ ".

After the experiment by Friedman and Rainwater, it became clear that the limit on $R$ could only be lowered by counter experiments. The first experiment of this type was performed by Lokanathan and Steinberger ${ }^{23}$ in 1954 using the apparatus shown in Fig. 1. A $\pi^{+}$beam from the Nevis synchrocyclotron was brought to rest in a thin $\mathrm{CH}_{2}$ target and decay positrons were detected by a scintillation counter hodoscope, which contained a variable thickness of $\mathrm{CH}_{2}$ absorber. Most of the data were taken with an absorber of $23 \mathrm{~cm}$ in thickness, which corresponds to an energy loss of 55 $\mathrm{MeV}$ from ionisation alone, above the end-point of $\mu^{+} \rightarrow \mathrm{e}^{+} \nu \bar{\nu}$ decay. However, some of these positrons could still traverse the telescope through the conversion of their bremsstrahlung. As a consequence, even with a $23-\mathrm{cm}$ thick absorber the telescope could see not only the $\sim 70 \mathrm{MeV}$ positrons from $\pi^{+} \rightarrow \mathrm{e}^{+} \nu$ decay, but also the tail of the much more frequent positrons from $\mu^{+} \rightarrow \mathrm{e}^{+} \nu \bar{\nu}$ decay. They 


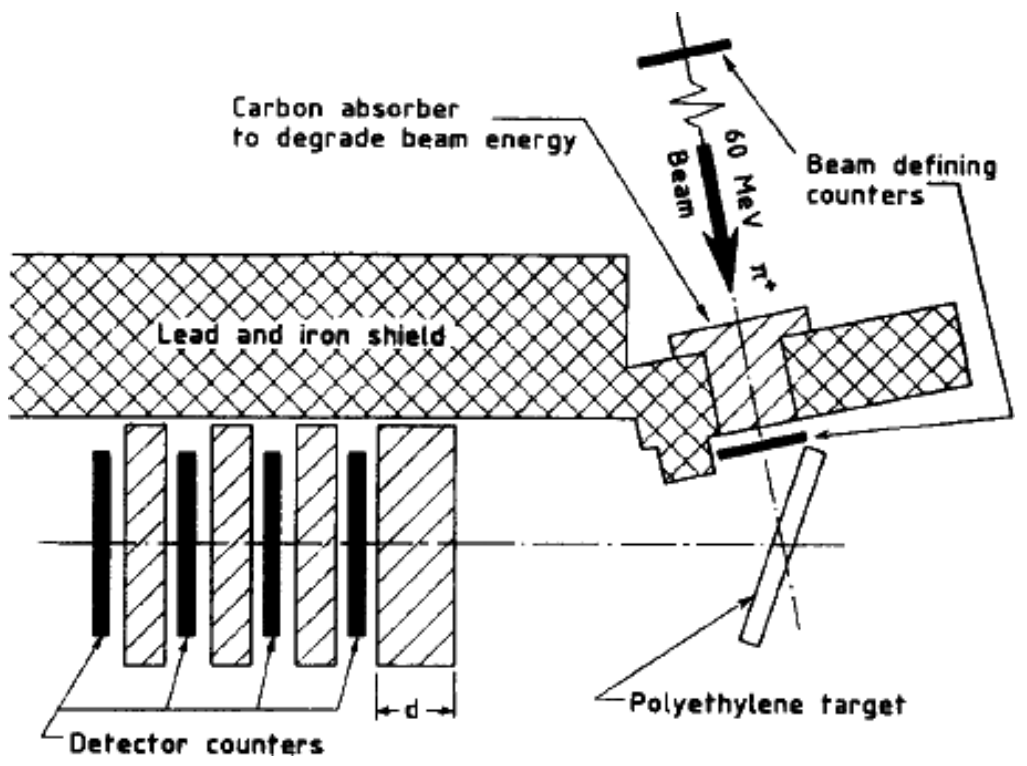

Fig. 1. Arrangement of counters and absorbers in the experiment of Lokanathan and Steinberger. ${ }^{23}$

measured $R=(-0.3 \pm 0.9) \times 10^{-4}$ and concluded: "It seems therefore improbable that the pion is coupled symmetrically to the muon."

I became interested in $\pi-\mathrm{e} \nu$ decay in the Summer 1954 at the Varenna Physics School in Varenna, Lake Como, Italy, where I attended a lecture by Steinberger on the first results from his experiment. This interest became stronger three years later, after attending a seminar at CERN by Herbert Anderson on June 12, 1957 on his search for $\pi-\mathrm{e} \nu$ decay together with Lattes. That experiment, published on December 1, 1957, ten years after the discovery of the pion, ${ }^{24}$ produced the most striking negative result. They used a magnetic spectrometer to measure the momentum distribution of electrons from stopped $\pi^{+}$-mesons (see Fig. 2). This technique was expected to provide a much higher rejection against electrons from $\mu^{+}$decay, thus being sensitive to $\pi^{+} \rightarrow \mathrm{e}^{+} \nu$ decays with $R$ values well below $10^{-4}$. The spectrometer was calibrated with $5.15 \mathrm{MeV} \alpha$-particles which have the same curvature as $98 \mathrm{MeV}$ positrons. Once again, no evidence for $\pi^{+} \rightarrow e^{+} \nu$ was found, giving $R=(-4.0 \pm 9.0) \times 10^{-6}$. The authors concluded: "This appears to be statistically significant and thereby allows only a $1 \%$ probability that $R$ could be greater than $2.1 \times 10^{-5}$."

After hearing the Chicago result, I had more discussions on this subject with Fazzini and Merrison, who were also interested in the subject, but we considered the possibility of doing an experiment rather remote at that time. 


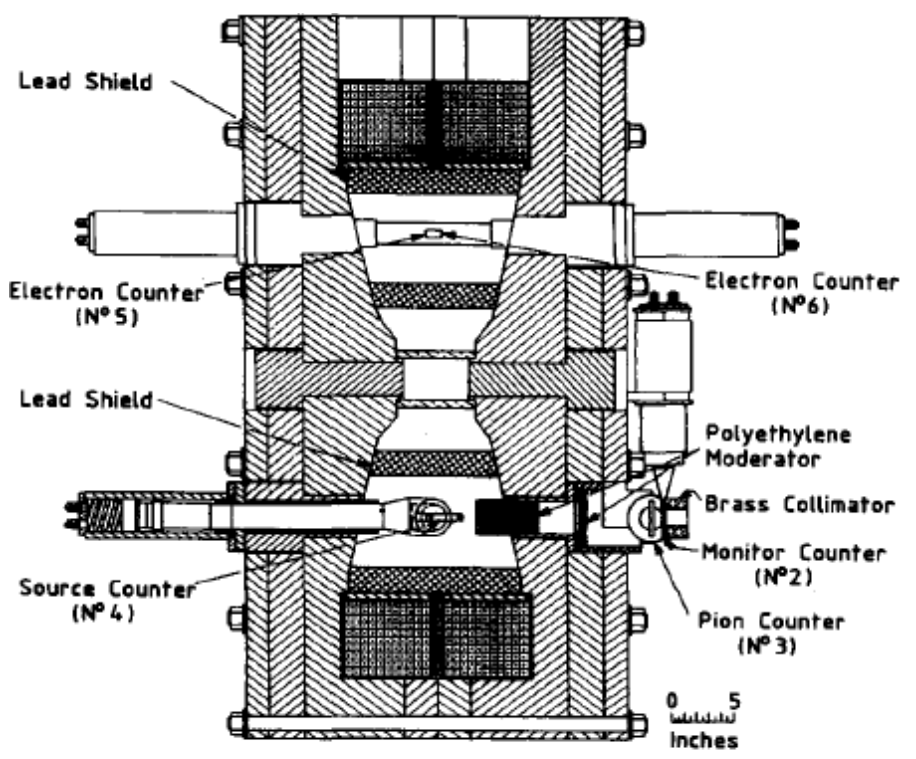

(a)

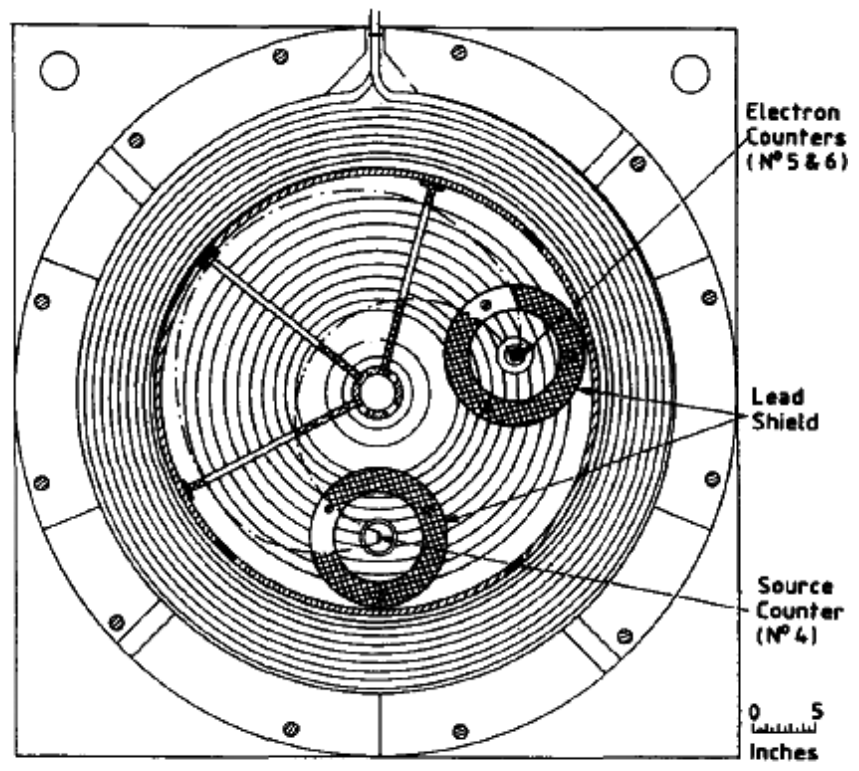

(b)

Fig. 2. Sectional view of the Chicago spectrometer. ${ }^{24}$ (a) Section along the incident beam direction. Pions are injected from the right parallel to the magnetic field direction through the brass collimator and come to rest in Counter 4. (b) Section through the median plane normal to the magnetic field direction. 
In the second half of 1957, three satisfactory formulations of the Universal Fermi Interaction were proposed almost at the same time:

- On July 16, Feynman and Gell-Mann submitted their famous paper "Theory of the Fermi interaction", proposing a universal V-A form. ${ }^{25}$ In this paper one could read "Experimentally no $\pi-\mathrm{e} \nu$ have been found, indicating that the ratio (to $\pi-\mu \nu$ ) is less than $10^{-5}$. This is a very serious discrepancy. The authors have no idea on how it can be resolved." And then at the end of the paper they concluded: "These theoretical arguments seem to the authors to be strong enough to suggest that the disagreement with the ${ }^{6} \mathrm{He}$ recoil experiment and with some other less accurate experiments indicates that these experiments are wrong. The $\pi-\mathrm{e} \nu$ problem may have a more subtle solution."

- On September 22-28, the International Conference on Mesons and Recently Discovered Particles took place in Padova and Venice. At this conference, Sudarshan and Marshak presented the paper "The Nature of the Four-Fermion Interaction" 26 in which they expressed doubts on the validity of the results from some experiments, on the searches for $\pi-\mathrm{e} \nu$ decay, in particular.

- On October 31, Sakurai submitted a paper "Mass Reversal and Weak Interactions" 27 along the same lines.

Sudarshan and Marshak stressed that although a mixture of vector and axial vector was the only universal four-fermion interaction that was possible and at the same time possessed many elegant features, it appeared that several published and unpublished experiments could not be reconciled with that hypothesis. They listed four experiments that had to be redone. Should any of the four experiments be confirmed, it would be necessary to abandon the hypothesis of a universal $\mathrm{V}-\mathrm{A}$ four-fermion interaction or at least one of the assumptions of a two-component neutrino and the conservation of leptons.

However, the suggestion of redoing the quoted experiments did not go too far because the Proceedings of the Padova-Venice Conference were only published in mid-1958 due to technical difficulties. Also, despite our interest in pion experiments, all of us (Fazzini, Merrison and myself) missed Sudarshan's talk in Venice because it was delivered in a session devoted to strange particles chaired by Heisenberg. We only knew of Sudarshan's suggestions several years later.

Nevertheless, the interest raised by the above papers called the attention to the experimental difficulties. The ${ }^{6} \mathrm{He}$ recoil experiment, namely the measurement of the electron-neutrino angular correlation in the decay ${ }^{6} \mathrm{He} \rightarrow{ }^{6} \mathrm{Li}+\mathrm{e}^{-}+\bar{\nu},{ }^{28}$ which required Tensor (T) coupling, appeared as a very serious difficulty. Even more serious appeared the absence of $\pi-\mathrm{e} \nu$ decay. There were perhaps already encouraging signals in the air that the ${ }^{6} \mathrm{He}$ recoil experiment had to be redone anyhow, as it looked like from a post-deadline paper presented at the New York meeting of the American Physical Society (APS) in January 1958, but that was certainly not the case for the absence of the $\pi-\mathrm{e} \nu$ decay. 
These problems were solved in 1958 by the first experiment performed at CERN with particles from a CERN accelerator. It was a search for $\pi^{+} \rightarrow \mathrm{e}^{+} \nu$ decay. ${ }^{29}$ That experiment, which "put CERN on the map of high energy physics", ${ }^{30}$ started the great CERN tradition of experimental work in weak interaction physics. It is remembered as a European success, but also as a success of the $600 \mathrm{MeV}$ CERN $\mathrm{SC}$, a machine rightly conceived to start research in Europe as early as possible before the $26 \mathrm{GeV}$ proton synchrotron was ready.

\section{3. $\pi$-Meson Decay to Electron and Neutrino: A CERN Discovery}

The paper by Feynman and Gell-Mann ${ }^{25}$ proposing a universal V-A form for the weak interaction was published on January 1, 1958. Unaware of that paper, I was attending the January 1958 meeting of the APS in New York, where I heard about the $\mathrm{V}-\mathrm{A}$ theory directly from Feynman who was giving an invited talk at that meeting. In the first part of that talk Feynman brilliantly described the $\mathrm{V}-\mathrm{A}$ theory and its successes. Then, in the second part he presented some ideas on how to strongly suppress $\pi \rightarrow \mathrm{e} \nu$ with respect to $\pi \rightarrow \mu \nu$ decay, probably an anticipation of the invited talk "Forbidding of $\pi-\beta$ decay" that he gave in the Summer of the same year at the International Conference on High-Energy Physics at CERN. ${ }^{31}$ In his talk Feynman was arguing that $\pi \rightarrow \mathrm{e} \nu$ decay might be strongly suppressed by the effect of large radiative corrections canceling the leading-order weak decay amplitude.

Feynman's arguments were not convincing, because radiative corrections were known to produce only effects at the few percent level in other processes. It was on that occasion that I decided — rightly or wrongly - to start a search for $\pi^{+} \rightarrow \mathrm{e}^{+} \nu$ decay at the CERN SC with a detector as simple as possible, without a spectrometer magnet, by stopping $\pi^{+}$-mesons in a scintillation counter and displaying the signals from this counter on the screen of an oscilloscope. This electronic system could be applied to an arrangement of counters and absorbers similar to that used by Lokanathan and Steinberger, ${ }^{23}$ where, however, $\pi^{+}$-mesons were stopped in an inert $\mathrm{CH}_{2}$ target, a crucial difference between the two experiments.

Such an experiment could be set up fairly quickly and also produce results quickly, but there would be a risk of failure should the $\pi \rightarrow \mathrm{e} \nu$ decay not exist at all. Or else it might not be approved because of the absence of a magnetic spectrometer. The general belief that the $\pi \rightarrow \mathrm{e} \nu$ decay either did not exist at all or its rate was much smaller than the theoretical prediction was so strong that very few people in a position of responsibility would have approved such an experiment (no committee would approve today an experimental search which, at least on paper, has a lower sensitivity than previous experiments that gave a null result).

In previous experiments, separation between electrons from $\pi^{+} \rightarrow \mathrm{e}^{+} \nu$ decays and from the $\pi^{+} \rightarrow \mu^{+} \rightarrow \mathrm{e}^{+}$decay chain had been achieved by measuring the decay electron energy, either by total absorption counters or by magnetic deflection. The main background of electrons from the $\pi^{+} \rightarrow \mu^{+} \rightarrow \mathrm{e}^{+}$decay chain at 


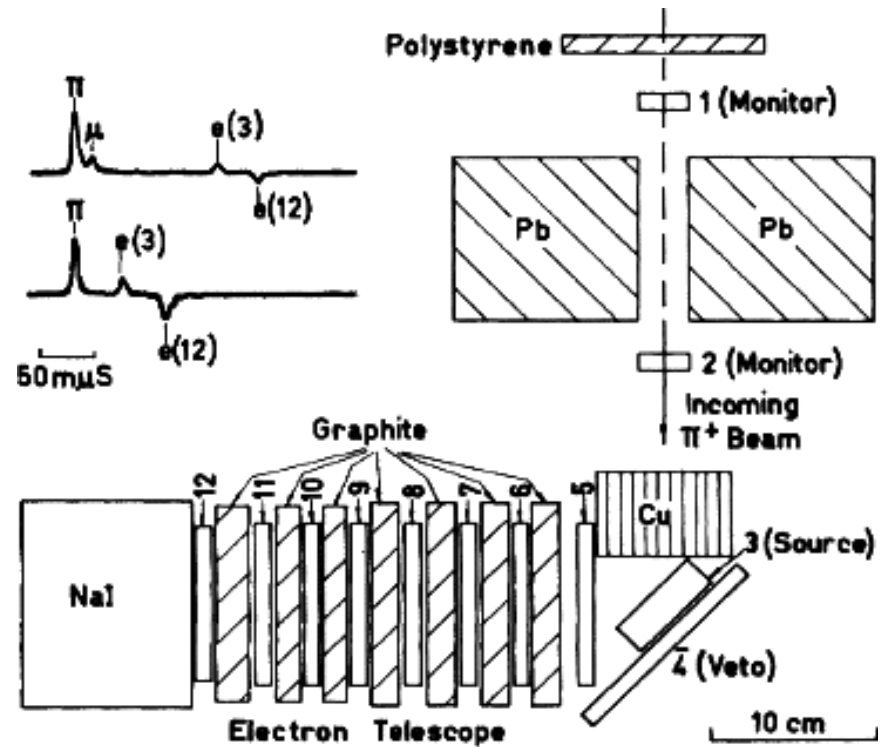

Fig. 3. Layout of the SC experiment ${ }^{29}$ together with typical $\pi^{+} \rightarrow \mu^{+} \rightarrow \mathrm{e}^{+}$and $\pi^{+} \rightarrow \mathrm{e}^{+}$ signals, as recorded on a fast oscilloscope (the time scale unit, "milli-micro-second" ( $\mathrm{m} \mu \mathrm{s})$ is called "nanosecond" (ns) today). Counter 3 is the active target where incident $\pi^{+}$mesons stop. The NaI counter information was not used in the final analysis.

rest has a maximum energy of $\sim 53 \mathrm{MeV}$ while electrons from $\pi^{+} \rightarrow \mathrm{e}^{+} \nu$ decay have an energy of $\sim 70 \mathrm{MeV}$. In the experiment of Lokanathan and Steinberger, ${ }^{25}$ the energy measurement method was adopted using a variable thickness counter telescope.

The SC experiment was performed in 1958 by Fazzini, Fidecaro, Merrison, Paul and Tollestrup. ${ }^{29}$ Work to set up the detector started in February, its construction was completed in May and the first run with beam took place on June 23.

The detector layout is shown in Fig. 3. Although the arrangement of counters and absorbers was similar to that of the experiment by Lokanathan and Steinberger ${ }^{23}$ (Fig. 1), the methods to recognise $\pi^{+} \rightarrow \mathrm{e}^{+} \nu$ decays from the $\pi^{+} \rightarrow \mu^{+} \rightarrow \mathrm{e}^{+}$decay chain in the $\mathrm{SC}$ experiment were completely different because, as mentioned earlier, in the SC experiment the $\pi^{+}$-mesons were stopped in an active target, namely in a plastic scintillator optically coupled to a photomultiplier.

The decay electron following a $\pi^{+}$stop, no matter from $\pi^{+}$or $\mu^{+}$decay, opened a gate whose length was equal to the length of the oscilloscope trace. The gate opened the door to the $\pi^{+}$stop signal (properly delayed) that in turn started the oscilloscope trace. The latter was always started at the same time with respect to the $\pi^{+}$stop signal, which, however, was shifted forward by an appropriate delay, so as to allow the inspection of the trace also before the arrival of the primary $\pi^{+}$. Figure 3 displays two typical traces. The upper one corresponds to a $\pi^{+} \rightarrow \mu^{+} \rightarrow \mathrm{e}^{+}$ decay event, with the second signal after the $\pi^{+}$stop associated with the muon from 
$\pi^{+} \rightarrow \mu^{+}$decay, and the third one, labelled e(3) associated with the positron from $\mu^{+} \rightarrow \mathrm{e}^{+}$decay. In the lower trace, there are only two signals from Counter 3 , with no intermediate $\mu^{+}$signal between the two, as expected from a $\pi^{+} \rightarrow \mathrm{e}^{+}$decay event. In both traces, the signal labelled e(12) is obtained from Counter 12, after a suitable delay, and is present on the trace together with signal e(3) only if the electron has traversed all the graphite absorbers and reached Counter 12.

The trace length covered a few $\pi^{+}$lifetimes after the $\pi^{+}$stop signal but only a fraction of the $\mu^{+}$lifetime (the $\pi^{+}$lifetime is $\sim 26 \mathrm{~ns}$, while the $\mu^{+}$lifetime is $\sim 80$ times longer). Under normal running conditions, the rate at which the oscilloscope trace started was only a few per hour.

In the experiment of Lokanathan and Steinberger ${ }^{23}$ the signal from the incoming $\pi^{+}$could still be used, but the presence of the intermediate $\mu^{+}$in the $\pi^{+} \rightarrow \mu^{+} \rightarrow \mathrm{e}^{+}$ decay chain could not be detected because the $\mu^{+}$remained invisible in the inert $\mathrm{CH}_{2}$ target (the $\mu^{+}$range from $\pi^{+} \rightarrow \mu^{+}$decay at rest is less than $1 \mathrm{~mm}$ in $\mathrm{CH}_{2}$ ).

Figure 4 is a photograph of the target region of the SC experiment, while Fig. 5 shows the fast oscilloscope and associated camera that were used to record the signals from the scintillator where the incident $\pi^{+}$-mesons were stopped. Finally, the racks containing the electronics in the counting room of the of the $\mathrm{SC}$ experiment are shown in Fig. 6.

In the choice of the method used in the SC experiment ${ }^{29}$ to distinguish $\pi^{+} \rightarrow$ $\mathrm{e}^{+} \nu$ decays from the $\pi^{+} \rightarrow \mu^{+} \rightarrow \mathrm{e}^{+}$decay chain, I was influenced by the single photomultiplier experiment of Janes and Kraushaar ${ }^{32}$ performed in 1953 at the M.I.T. $300 \mathrm{MeV}$ electron synchrotron to measure the photoproduction cross-section

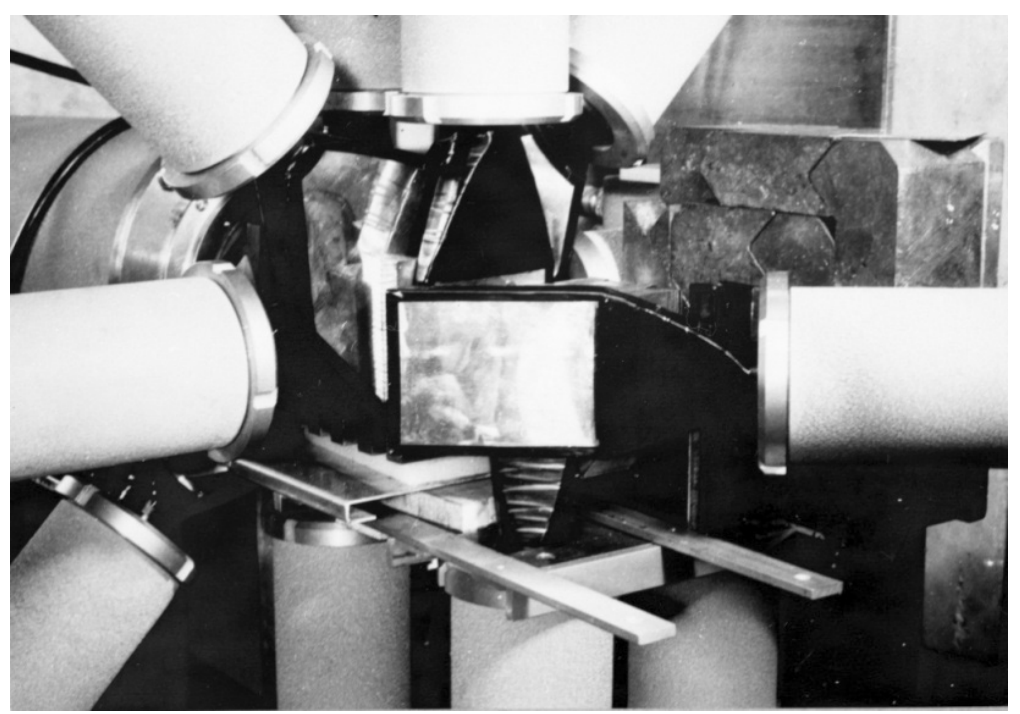

Fig. 4. Counter arrangement in the target region of the SC experiment. ${ }^{29}$ 


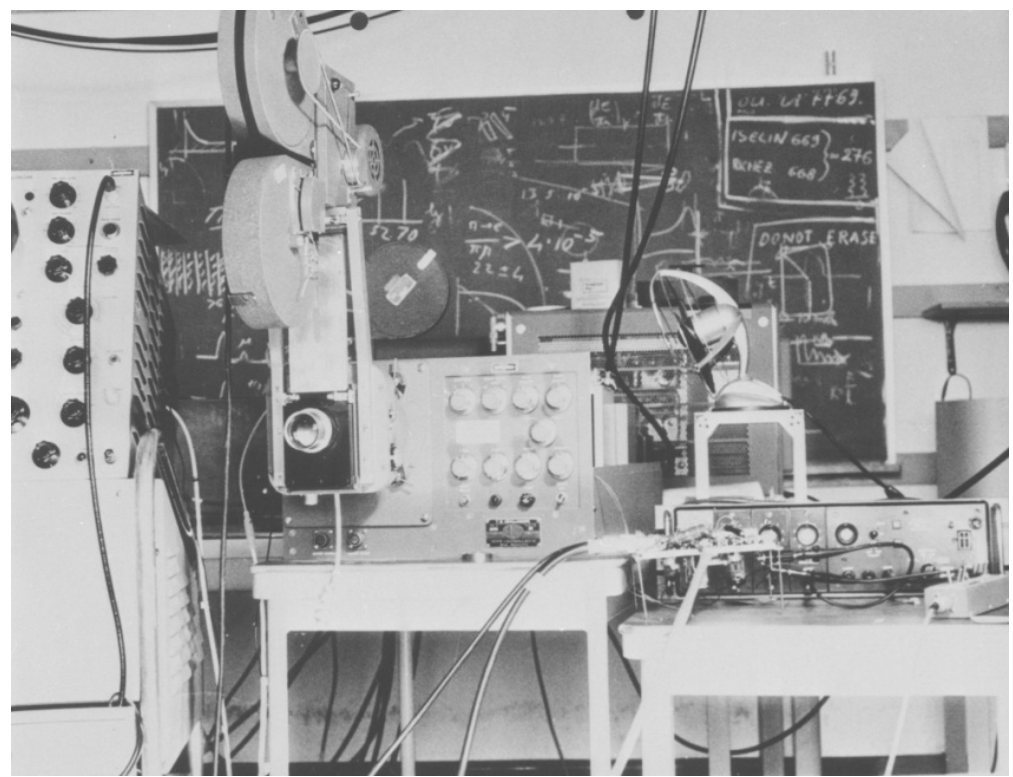

Fig. 5. The travelling-wave fast oscilloscope equipped with a photographic camera used in the $\mathrm{SC}$ experiment to record the target signals. ${ }^{29}$ On the blackboard one can see hand-written notes from a discussion on the first results, including a preliminary lower limit on $R$, suggesting that this photograph was taken at the end of August 1958.

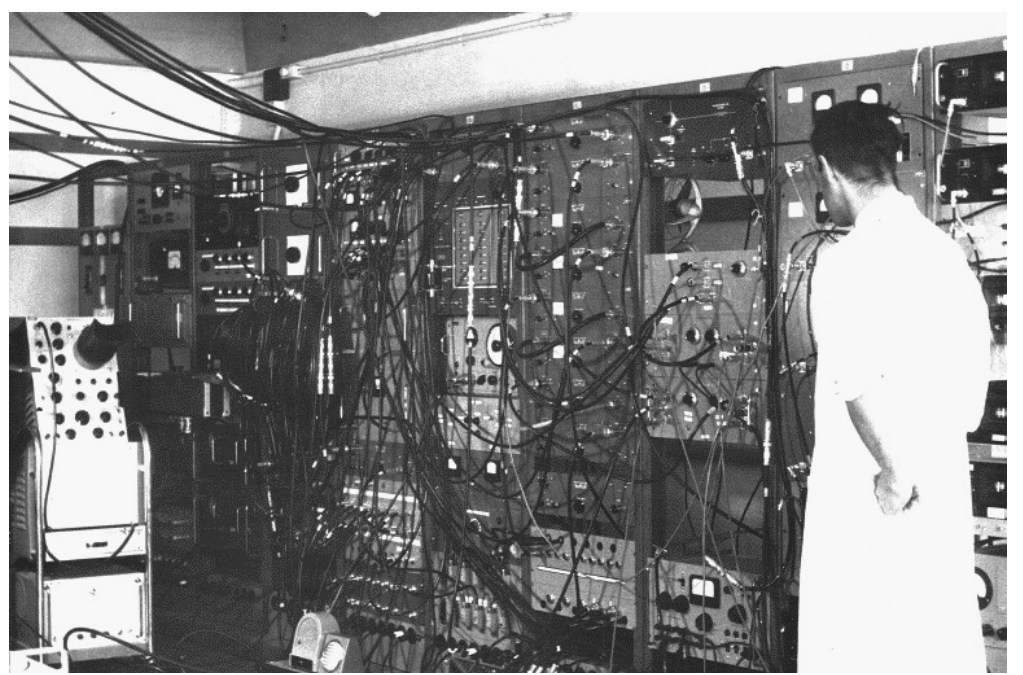

Fig. 6. The main electronic racks in the counting room of the $\mathrm{SC}$ experiment. ${ }^{29}$ 
of $\pi^{+}$-mesons from hydrogen and carbon at $90^{\circ}$, down to $10 \mathrm{MeV}$. To identify the $\pi^{+}$-mesons against a background of stable particles, they exploited the unique property of $\pi^{+}$-decays at rest in a scintillator by measuring the two consecutive signals from $\pi^{+} \rightarrow \mu^{+}$decay on the trace of a fast oscilloscope.

\subsection{Results}

Figure 7 shows the first results of this experiment in which the electron rate is presented as a function of the absorber thickness for both $\pi^{+} \rightarrow \mu^{+} \rightarrow \mathrm{e}^{+}$events and $\pi^{+} \rightarrow \mathrm{e}^{+}$candidates. The contamination of false $\pi^{+} \rightarrow \mathrm{e}^{+} \nu$ decays, i.e., $\pi^{+} \rightarrow \mu^{+} \rightarrow \mathrm{e}^{+}$events with the $\mu^{+}$signal in the target too near in time to the $\pi^{+}$signal to be resolved from it, was directly measured with small absorber thickness, where the electron rate is dominated by $\pi^{+} \rightarrow \mu^{+} \rightarrow \mathrm{e}^{+}$decays. A total of 40 candidates of $\pi^{+} \rightarrow \mathrm{e}^{+} \nu$ decay were observed with an absorber thickness of 30 to $34 \mathrm{~g} / \mathrm{cm}^{2}$, to be compared with an expected number of four false $\pi^{+} \rightarrow \mathrm{e}^{+} \nu$ decays. The time distribution of the positrons in the 40 candidates had an exponential form with a decay constant $\tau=22 \pm 4 \mathrm{~ns}$, consistent with the known $\pi^{+}$ lifetime.

Here it should be stressed that, contrary to the experiment by Lokanathan and Steinberger, ${ }^{23}$ the selection of $\pi^{+} \rightarrow \mathrm{e}^{+} \nu$ events was based on the presence of only two signals on the oscilloscope trace, and not on the absorber thickness traversed by the positron. On hindsight, the experiment could have been done with a simpler electron telescope consisting of only two counters with a variable absorber in between.

These results became available only after the 1958 International Conference on High-Energy Physics at CERN. I presented them for the first time on Thursday, September 4, 1958, at an informal session on Fundamental and High Energy Physics of the 2nd United Nations International Conference on the Peaceful Uses of Atomic Energy, which took place in Geneva on September 1-13, 1958. The session was chaired by Weisskopf and the Scientific Secretaries were I. Ulehla and A. Salam. The audience was rather small, only two or three dozens of people, contrary to other sessions with gigantic audience. Only three speakers were on the session programme. Feynman, particularly interested in the subject of my presentation, was in the audience. The results of the CERN experiment were quoted by several invited speakers and in the closing talk of the conference. ${ }^{33}$

The results shown in Fig. 7 represent the first experimental evidence for the decay $\pi^{+} \rightarrow \mathrm{e}^{+} \nu$. In order to derive a value of $R$, it was necessary to estimate the positron detection efficiency (this was not trivial at that time because electronic computers were still in their infancy). Lacking a precise knowledge of this efficiency, the observation gave the lower limit $R>4 \times 10^{-5},{ }^{29}$ consistent with the $\mathrm{V}-\mathrm{A}$ expectation. 


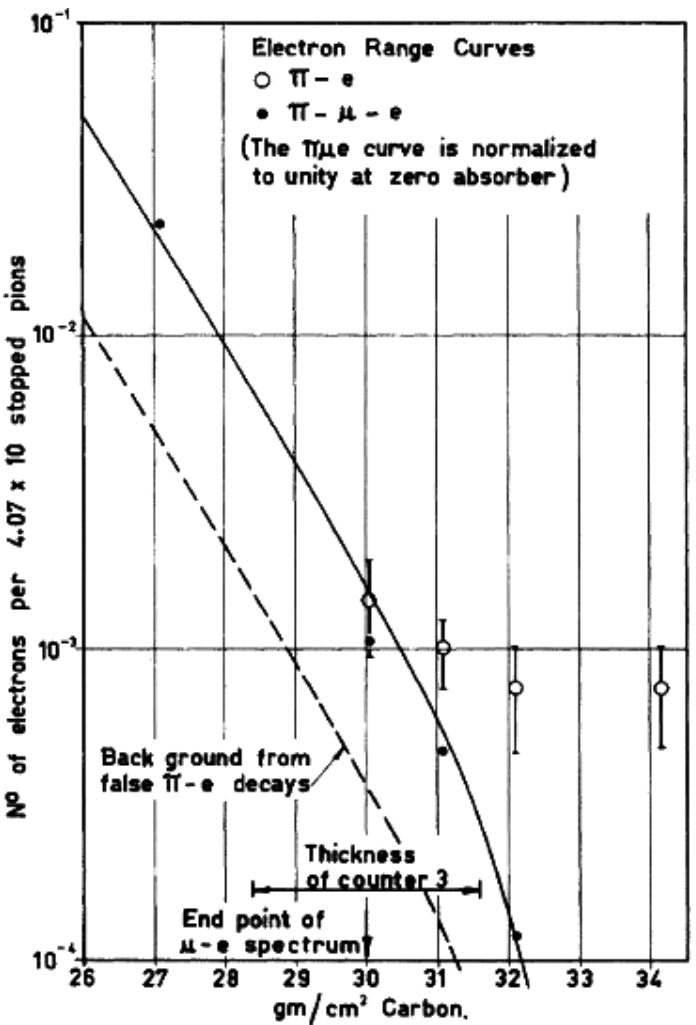

Fig. 7. Range curves for $\pi-\mu-\mathrm{e}$ (full circles) and $\pi-e$ (open circles), as measured in the SC experiment. $^{29}$ The full curve is a smooth line through the $\pi-\mu-$ e points. The dashed line is the range curve for unresolved $\pi-\mu-$ e events, as obtained from runs with no absorber in the electron telescope. The fraction of unresolved $\pi-\mu-\mathrm{e}$ events was 0.23 of the total detected number of $\pi-\mu-\mathrm{e}$ events.

At the end of 1958, Julius Ashkin from Carnegie-Mellon joined the group and gave important contributions to the calculation of the positron detection efficiency by developing a Monte Carlo program to this purpose. These calculations were first done in Rome using the computer of the National Research Council (C.N.R.) and then at CERN, when the first electronic computer (a Britishmade Ferranti Mercury computer) was installed. The final paper, published in 1959, includes the results from these calculations, giving the result $R=$ $(1.22 \pm 0.30) \times 10^{-4}, 34$ which is in excellent agreement with the electron-muon universality of the A coupling and in disagreement with the result of the Chicago experiment. $^{24}$

This result was soon confirmed by the Columbia group, ${ }^{35}$ who found evidence for $\pi^{+} \rightarrow \mathrm{e}^{+} \nu$ decay from a re-analysis of $65,000 \pi^{+}$stops in a liquid hydrogen bubble chamber operating in a magnetic field of $0.88 \mathrm{~T}$. 
It is amusing to compare the 1958 measurement of $R$ at the $\mathrm{SC}$ with the present world average, ${ }^{36} R=(1.230 \pm 0.004) \times 10^{-4}$.

\section{First Observation of the Decay $\pi^{+} \rightarrow \pi^{0} \mathrm{e}^{+} \nu$}

A further important experiment performed at the SC in 1962 achieved the first measurement of the pion beta decay mode $\pi^{+} \rightarrow \pi^{0} \mathrm{e}^{+} \nu$. This provided an excellent confirmation of the theory since the rate of this decay can be reliably predicted. It is a $0^{-}-0^{-}$transition between two levels of an isotopic triplet and thus a "superallowed" pure Fermi transition. The strength of such transitions is known from nuclear beta decays and, after correcting for the different phase space, the decay rate could be predicted as $\Gamma\left(\pi^{+} \rightarrow \pi^{0} \mathrm{e}^{+} \nu\right)=(0.393 \pm 0.002) \mathrm{s}^{-1}$, which corresponds to a very small branching ratio, $\mathrm{B}\left(\pi^{0} \mathrm{e}^{+} \nu\right)=1.02 \times 10^{-8}$.

The first observation of this rare decay mode was made at the $\mathrm{SC}^{37}$ by Depommier, Heintze, Mukhin, Rubbia, Sörgel and Winter using the apparatus shown in Fig. 8 (in the group, Mukhin was a visiting scientist from JINR, Dubna, USSR). A $\pi^{+}$-beam was brought to rest in a scintillation counter that served to

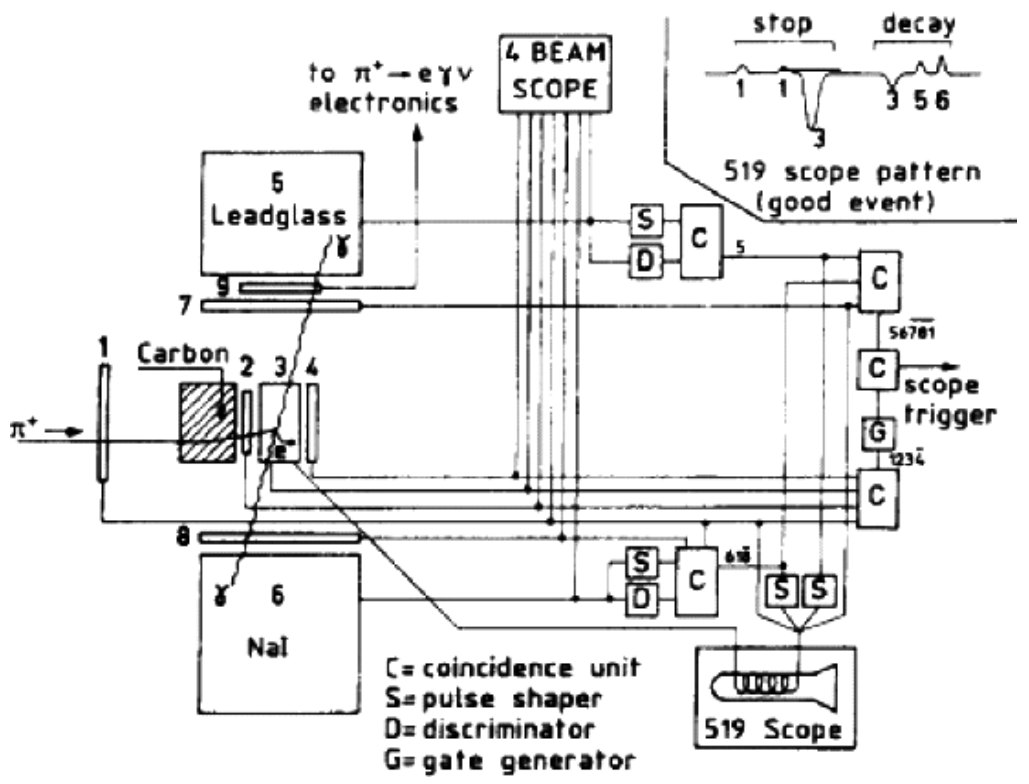

Fig. 8. Counter arrangement and electronics diagram in the first SC experiment which measured the $\pi^{+} \rightarrow \pi^{0} \mathrm{e}^{+} \nu$ decay rate. ${ }^{37,38}$ The pattern of signals recorded on a fast oscilloscope is shown in the upper right corner. 


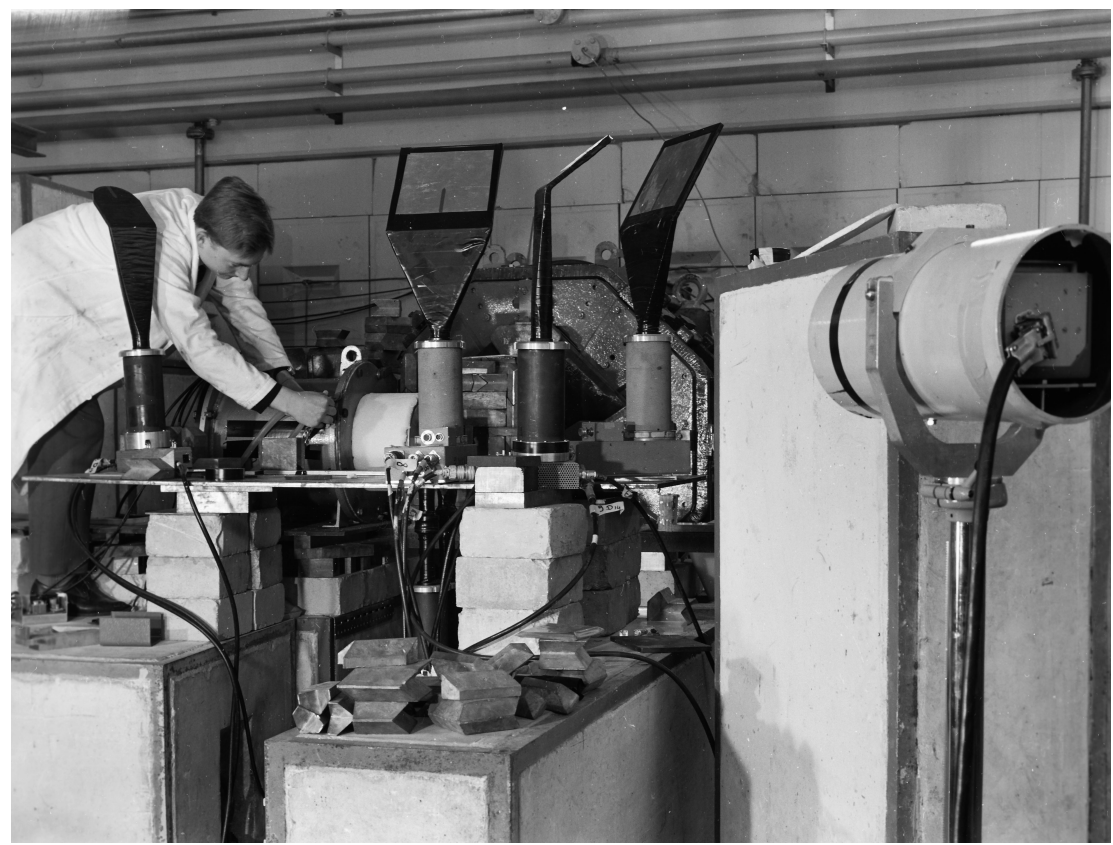

Fig. 9. The SC experiment to measure the $\pi^{+} \rightarrow \pi^{0} \mathrm{e}^{+} \nu$ decay rate ${ }^{37,38}$ during installation.

detect the decay $\mathrm{e}^{+}$and to measure its energy from pulse height (the maximum $\mathrm{e}^{+}$ energy from $\pi^{+} \rightarrow \pi^{0} \mathrm{e}^{+} \nu$ decay is $4.5 \mathrm{MeV}$, including the contribution from $\mathrm{e}^{+} \mathrm{e}^{-}$ annihilation in the counter). The two photons from $\pi^{0}$ decay, which are emitted with an opening angle always greater than $176^{\circ}$, were detected by a NaI crystal and a lead-glass Cherenkov counter in coincidence. A photograph of the apparatus during installation is shown in Fig. 9.

In a first run 16 candidates of $\pi^{+} \rightarrow \pi^{0} \mathrm{e}^{+} \nu$ decay were observed with an estimated background of $2.0 \pm 1.3$ events, ${ }^{37}$ giving a branching ratio $\mathrm{B}\left(\pi^{0} \mathrm{e}^{+} \nu\right)=$ $(1.7 \pm 0.5) \times 10^{-8}$. Additional data-taking increased the event sample to 44 candidates with an estimated background of $6 \pm 2$ events, ${ }^{38}$ corresponding to a branching ratio $\mathrm{B}\left(\pi^{0} \mathrm{e}^{+} \nu\right)=(1.15 \pm 0.22) \times 10^{-8}$, which is in good agreement with the theoretical prediction.

A second experiment performed a few years later ${ }^{39}$ with a lead-glass photon spectrometer having a much larger angular coverage (see Fig. 10) provided a sample of 411 candidates with an estimated background of $79 \pm 10$ events. This gave the branching ratio $\mathrm{B}\left(\pi^{0} \mathrm{e}^{+} \nu\right)=\left(1.00_{-0.10}^{+0.08}\right) \times 10^{-8}$, confirming the theoretical prediction at the $10 \%$ level.

The present world average is ${ }^{36} \mathrm{~B}\left(\pi^{0} \mathrm{e}^{+} \nu\right)=(1.036 \pm 0.006) \times 10^{-8}$. 


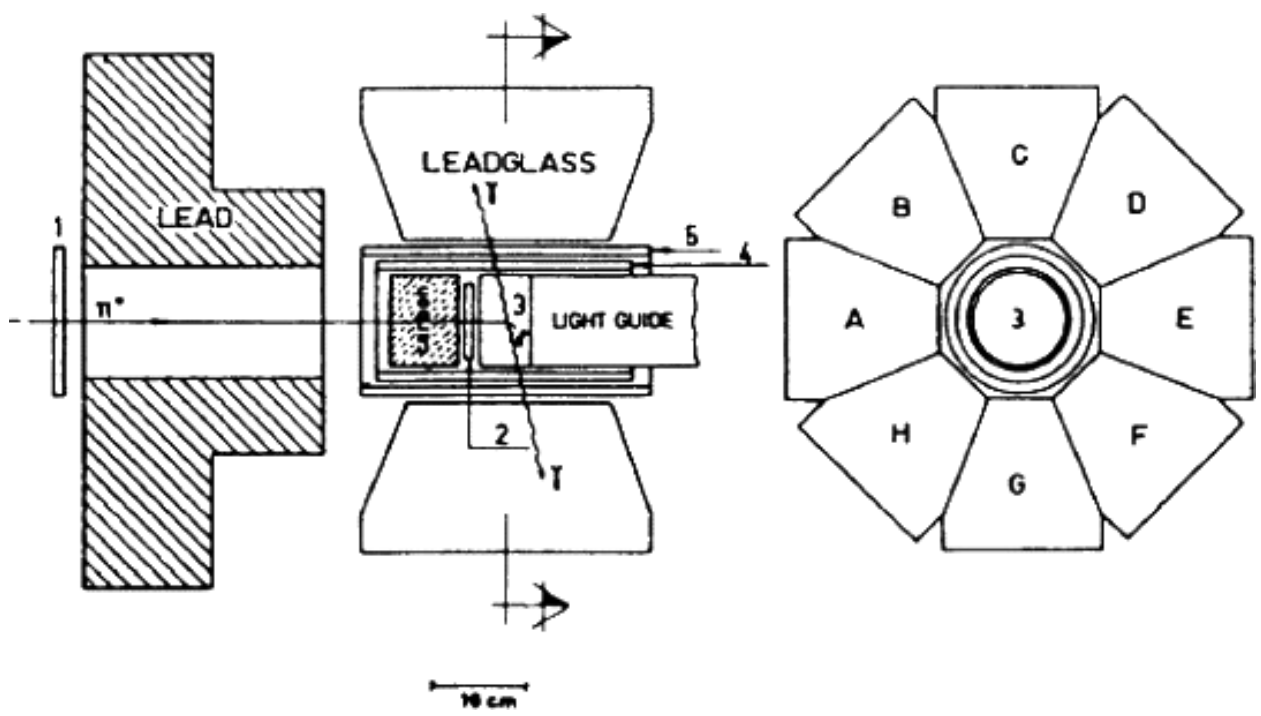

Fig. 10. Counter arrangement of the second SC measurement ${ }^{39}$ of the $\pi^{+} \rightarrow \pi^{0} \mathrm{e}^{+} \nu$ decayrate.

\section{Conclusions}

The SC was the first accelerator to be built at CERN, with the main purpose of providing an opportunity for European physicists to learn how to do high energy physics. It began operation several years after other machines of similar energy and intensity, such as the synchrocyclotrons at Berkeley, Dubna, Chicago, Liverpool or Nevis. Nevertheless, it made remarkable contributions to particle physics, among which are the results of historical importance on $\pi^{+} \rightarrow \mathrm{e}^{+} \nu$ and $\pi^{+} \rightarrow \pi^{0} \mathrm{e}^{+} \nu$ decay described in this article.

Other important particle physics experiments at the SC include: ${ }^{40}$

- Searches for $\mu \rightarrow \mathrm{e} \gamma$ decay and neutrinoless $\mu^{-}$-capture, whose negative results pointed to the existence of a second neutrino;

- The measurement of the positron helicity from $\mu^{+}$-decay;

- Measurements of the $\mu^{-}$capture rate in hydrogen, both in liquid and gaseous form;

- Last but not least, the first measurement of the muon anomalous magnetic moment, described in Farley's article of this book. ${ }^{41}$

From its spectacular start to its closing down in 1990, the SC has considerably contributed to the scientific reputation of CERN. 


\section{References}

1. T. Fazzini, G. Fidecaro and H. Paul, Nucl. Instr. 3, 156 (1959).

2. E. Fermi, Z. Phys. 88, 161 (1934) (in German); Nuovo Cim. 11, 1 (1934) (in Italian).

3. H. Yukawa, Proc. Math. Soc. Japan 17, 48 (1935).

4. C. Møller, L. Rosenfeld and S. Rozenthal, Nature 144, 629 (1939).

5. S. Sakata, Phys. Rev. 58, 576 (1940).

6. E. Fermi, Elementary Particles, p. 110, Yale University Press, New Haven (1951).

7. M. Conversi, E. Pancini and O. Piccioni, Phys. Rev. 71, 209 (1947).

8. B. Pontecorvo, Phys. Rev. 72, 246 (1947).

9. G. Puppi, Nuovo Cim. 5, 587 (1948).

10. C. N. Yang and J. Tiomno, Phys. Rev. 79, 495 (1950).

11. C. M. G. Lattes, G. P. S. Occhialini and C. F. Powell, Nature 160, 453 (1947).

12. E. Gardner and C. M. G. Lattes, Science 107, 270 (1948).

13. A. S. Bishop, J. Burfening, E. Gardner and C. M. G. Lattes, Phys. Rev. 74, 1558 (1948).

14. C. M. G. Lattes, Phys. Rev. 75, 1468 (1949).

15. J. Tiomno and J. A. Wheeler, Rev. Mod. Phys. 21, 153 (1949).

16. T. D. Lee, M. Rosenbluth and C. N. Yang, Phys. Rev. 75, 905 (1949).

17. M. Ruderman and R. Finkelstein, Phys. Rev. 76, 1458 (1949).

18. J. Steinberger, Phys. Rev. 76, 1180 (1949).

19. C. S. Wu and S. A. Moszkowski, Beta Decay, Interscience, New York, (1966), p. 239.

20. E. F. Pessôa and N. Margem, An. Acad. Brasil. Ciênc. 22, 371 (1950) (in Portuguese).

21. F. M. Smith, Phys. Rev. 81, 897 (1951).

22. H. L. Friedman and J. Rainwater, Phys. Rev. 81, 644 (1951); Phys. Rev. 84, 684 (1951).

23. S. Lokanathan and J. Steinberger, Suppl. Nuovo Cim. 2, 151 (1955).

24. H. L. Anderson and C. M. G. Lattes, Nuovo Cim. 6, 1356 (1957).

25. R. P. Feynman and M. Gell-Mann, Phys. Rev. 109, 193 (1958).

26. E. C. G. Sudarshan and R. E. Marshak, The nature of the four-fermion interaction, in Proc. Int. Conf. on Mesons and Recently Discovered Particles, Padova-Venezia (Italy), Sept. 22-28, 1957 (Borghero, Padova, 1958), p. V-14.

27. J. J. Sakurai, Nuovo Cim. 7, 649 (1958).

28. B. M. Rustad and S. L. Ruby, Phys. Rev. 97, 991 (1955).

29. T. Fazzini, G. Fidecaro, A. W. Merrison, H. Paul and A. V. Tollestrup, Phys. Rev. Lett. 1, 247 (1958).

30. J. J. Sakurai, The structure of charged currents, in Proc. Int. Conf. on Neutrino Physics and Astrophysics, Dept. of Physics and Astronomy, Honolulu (1981), Vol. 2, p. 457.

31. R. P. Feynman, Forbidding of $\pi-\beta$ decay, in Proc. Int. Conf. on High Energy Physics, CERN, Geneva, ed. B. Ferretti (1958) p. 216.

32. G. S. Janes and W. L. Kraushaar, Phys. Rev. 93, 900 (1954).

33. Proceedings of the $2^{\text {nd }}$ United Nations Int. Conf. on the Peaceful Uses of Atomic Energy (United Nations, Geneva, 1958), Vol. 1, p. 389; Vol. 30, pp. 42, 57, 136, 327-328. 
34. J. Ashkin, T. Fazzini, G. Fidecaro, A. W. Merrison, H. Paul and A. V. Tollestrup, Nuovo Cim. 13, 1240 (1959).

35. G. Impeduglia, R. Plano, A. Prodell, N. Samios, M. Schwartz and J. Steinberger, Phys. Rev. Lett. 1, 249 (1958).

36. K. A. Olive et al. (Particle Data Group), Chinese Physics C 38, 090001 (2014), see page 34 .

37. P. Depommier, J. Heintze, A. Mukhin, C. Rubbia, V. Sörgel and K. Winter, Phys. Lett. 2, 23 (1962).

38. P. Depommier, J. Heintze, C. Rubbia and V. Sörgel, Phys. Lett. 5, 61 (1963).

39. P. Depommier, J. Duclos, J. Heintze, K. Kleinknecht, H. Rieseberg and V. Sörgel, Nucl. Phys. B4, 189 (1968); Nucl. Phys. B4, 432 (1968).

40. L. Di Lella, Elementary particle physics at the SC, Phys. Repo. 225, 45 (1993).

41. F. J. M. Farley, Muon $g-2$ and Tests of Relativity, in this book, pp. 371-396. 